\title{
Two-Dimensional Drexhage Experiment for Electric- and Magnetic-Dipole Sources on Plasmonic Interfaces
}

\author{
Raphael Brechbühler, ${ }^{1}$ Freddy T. Rabouw, ${ }^{1}$ Patrik Rohner, ${ }^{2}$ Boris le Feber, ${ }^{1}$ \\ Dimos Poulikakos, ${ }^{2}$ and David J. Norris ${ }^{1, *}$ \\ ${ }^{1}$ Optical Materials Engineering Laboratory, ETH Zurich, 8092 Zurich, Switzerland \\ ${ }^{2}$ Laboratory of Thermodynamics in Emerging Technologies, ETH Zurich, 8092 Zurich, Switzerland
}

(Received 19 March 2018; published 12 September 2018)

\begin{abstract}
Fifty years ago, Drexhage et al. showed how photon emission from an electric dipole can be modified by a nearby mirror. Here, we study the two-dimensional analog for surface plasmon polaritons (SPPs). We print $\mathrm{Eu}^{3+}$-doped nanoparticles, which act as both electric- and magnetic-dipole sources of SPPs, near plasmonic reflectors on flat Ag films. We measure modified SPP radiation patterns and emission rates as a function of reflector distance and source symmetry. The results, which agree with an analytical selfinterference model, provide simple strategies to control SPP radiation in plasmonic devices.
\end{abstract}

DOI: 10.1103/PhysRevLett.121.113601

Surface plasmon polaritons (SPPs) are electromagnetic waves coupled to charge-density oscillations at a metaldielectric interface. Because they are strongly confined at this interface, SPPs allow for the manipulation of light at the nanoscale and enhancement of light-matter interactions. To exploit SPPs, they must first be generated, e.g., with near-field sources, such as quantum emitters [1-3], optical near-field probes [4], or scattering structures $[5,6]$. The plasmonic environment near these sources, e.g., nearby reflectors, slits, or scatterers, can be used to direct SPPs or enhance emission rates [3,7-9].

Typically, sources with electric-dipole (ED) character have been used, which couple to the electric component of electromagnetic waves. However, many sources of SPPs also exhibit magnetic-dipole (MD) character. For example, plasmonic structures, such as simple subwavelength holes [6,10] or slits [5], metallic split-ring resonators [11], and dielectric Mie scatterers [12], feature significant magnetic polarizability. In addition, certain emitters, such as transition-metal and lanthanide ions, can exhibit MD transitions at visible [13] and near-infrared frequencies $[14,15]$. Because of their distinct emission-field symmetries, we anticipate qualitative differences in how ED and MD sources of SPPs interact with the plasmonic environment, thus affecting the SPP radiation patterns and emission rates. However, studies demonstrating and exploiting these differences have been missing.

Published by the American Physical Society under the terms of the Creative Commons Attribution 4.0 International license. Further distribution of this work must maintain attribution to the author(s) and the published article's title, journal citation, and DOI.
In contrast, the interaction of photon sources with their optical environment has been studied in depth. Fifty years ago, Drexhage et al. reported variations of the spontaneous photon emission rate of ED sources placed at increasing distances from a mirror [16,17]. These findings can be explained by self-interference of the emitted light with its reflection from the mirror [17] or, equivalently, as a modification of the local density of optical states caused by the mirror [18]. Later, MD sources were shown to interact differently with the optical environment than ED sources due to their opposing emission-field symmetries [19-21]. A plasmonic analog of Drexhage's experiment would provide insights into source-SPP interaction with implications for directional single-SPP sources, focusing of SPPs onto hot spots, and enhancement or inhibition of SPP emission from quantum emitters. A realization of this plasmonic experiment requires nanoscopic sources of SPPs that are placed with subwavelength precision in front of a plasmonic reflector.

In this Letter, we experimentally demonstrate how MD and ED sources interact with their local plasmonic environment. Recent developments now make it possible to place SPP sources with ED and MD character in the vicinity of a plasmonic reflector on a flat $\mathrm{Ag}$ surface. This simple geometry is the two-dimensional equivalent of Drexhage's original experiment and serves as a model system for more complex plasmonic environments. Experimentally, we utilize $\mathrm{Eu}^{3+}$-doped nanocrystals as ED and MD emitters. Self-interference of the dipole-excited SPPs with their reflection at the plasmonic reflector leads to directional SPP radiation. We exploit the opposing field symmetries of EDs and MDs to channel their SPP emission into different directions. Further, we find oscillations of the relative SPP emission rates of the two dipole transitions as a function 
of the source-reflector distance. The experimental results are in excellent agreement with an analytical SPP selfinterference model.

We fabricated a structured plasmonic surface [Fig. 1(a)] consisting of a block reflector and quarter-circular outscatterer (details in the Supplemental Material [22]) by template stripping [26] a high-quality Ag film [27]. To provide local sources of SPPs, we deposited colloidal $\mathrm{NaYF}_{4}$ nanocrystals doped with $5 \% \mathrm{Eu}^{3+}\left(5 \%\right.$ of $\mathrm{Y}^{3+}$ from the $\mathrm{NaYF}_{4}$ host crystals replaced with $\mathrm{Eu}^{3+}$ ions; details in Ref. [22]) on the Ag surface with subwavelength precision at defined distances from the reflector using electrohydrodynamic nanoprinting [2,28]. This created $1-\mu \mathrm{m}$-long and $100-\mathrm{nm}$-wide lines parallel to the reflector, as shown in Fig. 1(b) and Supplemental Material Fig. S1 [22], with densely packed nanocrystals stacked on top of each other. The $\mathrm{Eu}^{3+}$ dopant ions exhibit multiple electronic transitions in the visible spectrum with narrow spectral linewidths (Supplemental Material Fig. S2 [22]). Here, we focus on two strong and closely spaced transitions from the common excited state ${ }^{5} D_{0}$ to the ground states ${ }^{7} F_{1}$ (centered at wavelength $\lambda=591 \mathrm{~nm})$ and ${ }^{7} F_{2}(\lambda=615 \mathrm{~nm})$
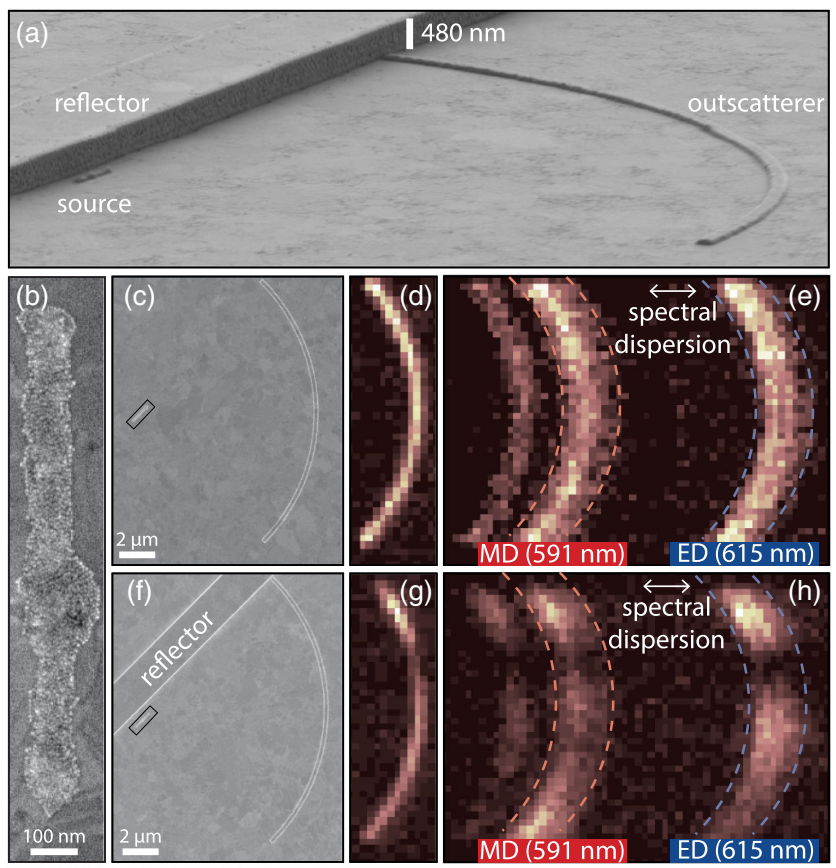

FIG. 1. Experimental setup. (a) SPP source on plasmonic Ag surface structured with 480-nm-tall reflector and quarter-circular outscatterer. (b) Close-up of the source consisting of a printed line of $\mathrm{NaYF}_{4}: \mathrm{Eu}^{3+}$ nanocrystals. (c)-(e) Nanocrystals (highlighted with black rectangle) placed $10 \mu \mathrm{m}$ from a quarter-circular outscatterer in absence of a plasmonic reflector. SPPs scattered into photons at the quarter-circle upon laser excitation of the nanocrystals collected as (d) real-space fluorescence image and (e) spectrally dispersed along the horizontal direction. (f)-(h) The corresponding experiment with a plasmonic reflector at $d=$ $544 \mathrm{~nm}$ from the nanocrystals. (a)-(c),(f) Electron micrographs. with MD and ED character, respectively [20]. The emitters are excited with a focused laser beam on the ${ }^{7} F_{0} \rightarrow{ }^{5} L_{6}$ transition at $395 \mathrm{~nm}$ using a $100 \times$ air objective (with numerical aperture, NA, 0.9). After fast nonradiative relaxation to the ${ }^{5} D_{0}$ excited state, decay to the ${ }^{7} F_{1}$ and ${ }^{7} F_{2}$ ground states can launch SPPs that travel along the flat Ag surface and outscatter at the quarter-circle. Outscattered photons are collected through the same objective and imaged on an electron-multiplying charge-coupled device camera [Figs. 1(d) and 1(g)]. To separate the emission stemming from the ED and MD transitions, the light is passed through a grating spectrometer [Figs. 1(e) and 1(h)].

First, we examine the coupling of the MD and ED transitions of $\mathrm{Eu}^{3+}$ to SPPs without a plasmonic reflector by placing emitters in the center of an outscattering quarter-circle as seen in Fig. 1(c). The signal intensity collected along the outscatterer is uniform [Fig. 1(d)]. Spectral dispersion of the signal [Fig. 1(e)] reveals that both the MD transition at $591 \mathrm{~nm}$ and the ED transition at $615 \mathrm{~nm}$ contribute to the scattering signal, meaning that in a homogeneous plasmonic environment both transitions emit SPPs isotropically. In contrast, when the nanocrystals are placed in front of a plasmonic reflector [Figs. 1(a) and 1(f)], a nonuniform signal is detected along the outscatterer [Fig. 1(g)]. In fact, a spectrally resolved image [Fig. 1(h)] shows that the SPP emission patterns from the MD and ED transitions are both anisotropic and different from each other. Clearly, the presence of a plasmonic reflector leads to distinct SPP emission patterns for the two types of dipole sources.

To understand the modification of the SPP emission, we consider the SPP emission patterns of ED and MD sources and their self-interference in the presence of a plasmonic reflector. $\mathrm{Eu}^{3+}$ ions are isotropic emitters [29]; i.e., their transition dipole moments sample all directions on timescales much shorter than the fluorescence lifetime [18]. The coupling of dipole sources to SPPs depends on the transverse-magnetic nature of the SPPs, which have their magnetic (electric) field oriented parallel (mostly perpendicular) to the metal-dielectric interface. Consequently, coupling is strongest to MD components oriented along and to ED components perpendicular to the interface. In addition, SPPs have a minor longitudinal electric-field component that couples to EDs oriented along the interface. However, we neglect this because it is $4 \times$ smaller than the transverse electric-field component

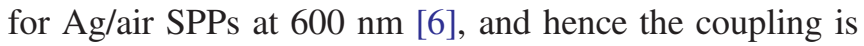
$16 \times$ weaker [22,30]. The SPP emission pattern [Fig. 2(a)] of the perpendicularly oriented ED component $p_{z}$ is isotropic [6]. The two parallel MD components $m_{x}$ and $m_{y}$ exhibit SPP emission patterns with electric fields proportional to $\sin \varphi$ and $\cos \varphi$ [6], respectively, where $\varphi$ is the in-plane angle with respect to the $x$ axis. Orientation-averaging then yields an isotropic MD intensity pattern. This explains the isotropic SPP emission patterns 


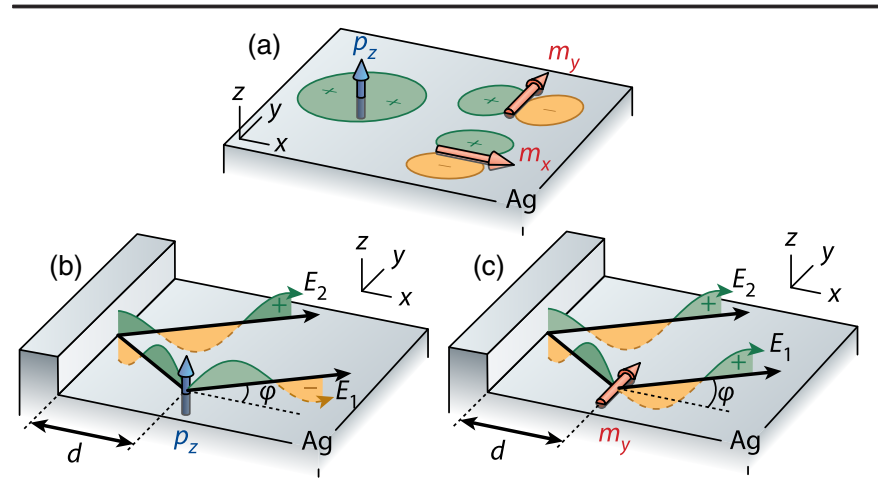

FIG. 2. (a) Electric-field-amplitude patterns of SPPs emitted by the dipole components $p_{z}, m_{x}$, and $m_{y}$ on a plasmonic substrate (xy plane) in absence of a reflector. Schematic representation of self-interference of the SPP emission from (b) a z-oriented ED and (c) a $y$-oriented MD in front of a plasmonic reflector.

for both the MD and ED transitions that we observe in the absence of a plasmonic reflector [Fig. 1(e)].

The plasmonic reflector introduces self-interference of the SPPs launched by MD and ED sources located at a separation $d$. At a large distance $R(\gg d)$ from the source, SPPs emitted directly in the positive $x$ direction [electric field $E_{1}$ depicted in Figs. 2(b) and 2(c)] interfere with SPPs reflected at the reflector [ $E_{2}$ in Figs. 2(b) and 2(c)] to yield a total emitted intensity $\left|E_{1}+E_{2}\right|^{2}$ depending on the emitterreflector separation $d$ and the in-plane SPP emission angle $\varphi$ (details in [22]). This intensity scales as

$$
\begin{aligned}
& I_{p_{z}}(d, \varphi) \propto\left|1+r(\varphi) \exp \left[i k_{\mathrm{SPP}} \Delta l(d, \varphi)\right]\right|^{2}, \\
& I_{m_{x}}(d, \varphi) \propto \sin ^{2} \varphi\left|1+r(\varphi) \exp \left[i k_{\mathrm{SPP}} \Delta l(d, \varphi)\right]\right|^{2},
\end{aligned}
$$

and

$$
I_{m_{y}}(d, \varphi) \propto \cos ^{2} \varphi\left|1-r(\varphi) \exp \left[i k_{\mathrm{SPP}} \Delta l(d, \varphi)\right]\right|^{2}
$$

for the ED component $p_{z}$, the MD component $m_{x}$, and the MD component $m_{y}$, respectively. The SPP wave vector $k_{\mathrm{SPP}}$ is given by the dispersion relation $k_{\mathrm{SPP}}=$ $k_{0} \sqrt{\varepsilon_{m} \varepsilon_{d} /\left(\varepsilon_{m}+\varepsilon_{d}\right)}$, where $k_{0}=2 \pi / \lambda$ is the free-space wave vector, and $\varepsilon_{d}=1$ and $\varepsilon_{m}$ are the relative permittivities of air and template-stripped $\mathrm{Ag}$ [27], respectively. $\Delta l=2 d \cos \varphi$ is the difference in path length traveled by SPPs $E_{1}$ and $E_{2}$. The reflection coefficient $r$ accounts for the phase shift and damping introduced upon reflection of the SPPs at the reflector. We approximate the value of $r$ using the Fresnel equations [31] for transverse-electric photons with wave vector $k_{\text {SPP }}$ incident on $\mathrm{Ag}$,

$$
r(\varphi)=\frac{\sqrt{k_{\mathrm{SPP}}^{2}-k_{\mathrm{SPP}}^{2} \sin ^{2} \varphi}-\sqrt{k_{0}^{2} \varepsilon_{m}-k_{\mathrm{SPP}}^{2} \sin ^{2} \varphi}}{\sqrt{k_{\mathrm{SPP}}^{2}-k_{\mathrm{SPP}}^{2} \sin ^{2} \varphi}+\sqrt{k_{0}^{2} \varepsilon_{m}-k_{\mathrm{SPP}}^{2} \sin ^{2} \varphi}} .
$$

Constructive self-interference occurs when the complex term $r \exp \left(i k_{\mathrm{SPP}} \Delta l\right)$ is real and positive for $p_{z}$ and $m_{x}$ or real and negative for $m_{y}$. The reason for the opposing conditions is the antisymmetric SPP emission pattern of dipole component $m_{y}$ about the $y$ axis, while the other two relevant dipole components show patterns that are mirror symmetric about the $y$ axis [Fig. 2(a)].

To model the SPP emission pattern of $\mathrm{Eu}^{3+}$ emitters at separation $d$ from a plasmonic reflector, we average over MD orientation to obtain $I_{\mathrm{MD}}=C\left(I_{m_{x}}+I_{m_{v}}\right)$ for the MD transition and $I_{\mathrm{ED}}=I_{p_{z}}$ for the $\mathrm{ED}$ transition. The scaling factor $C$ accounts for the difference in dipole strengths and coupling efficiencies to SPPs of the ${ }^{5} D_{0} \rightarrow{ }^{7} F_{1}$ and ${ }^{5} D_{0} \rightarrow{ }^{7} F_{2}$ transitions and has been experimentally measured in the absence of a reflector [Fig. 1(e)] to be $C \approx 0.79$. To account for the finite spatial width of the lines of $\mathrm{Eu}^{3+}$ doped nanocrystals in the experiments [Fig. 1(b) and Supplemental Material Fig. S1 [22] ], the calculated radiation patterns at distance $d$ are averaged over $d \pm 50 \mathrm{~nm}$ (see Supplemental Material Fig. S3 [22] for other choices).

In Figs. 3(a)-3(c), we present experimental (dots) and analytically calculated (lines) SPP emission patterns at different distances $d$ from the reflector (SPP emission patterns for all investigated $d$ and for transitions from the ${ }^{5} D_{1}$ excited state are shown in Supplemental Material Figs. S4 and S5 [22]). At $d=280 \mathrm{~nm}$ [Fig. 3(a); $d$ determined from scanning electron micrographs], the ED transition (blue) emits mainly at $\varphi \approx 60^{\circ}$, attributed to the first self-interference maximum of dipole component $p_{z}$. In contrast, the MD pattern (red) consists of two lobes, one at $\varphi=0^{\circ}$, where the ED emission reaches a minimum, and
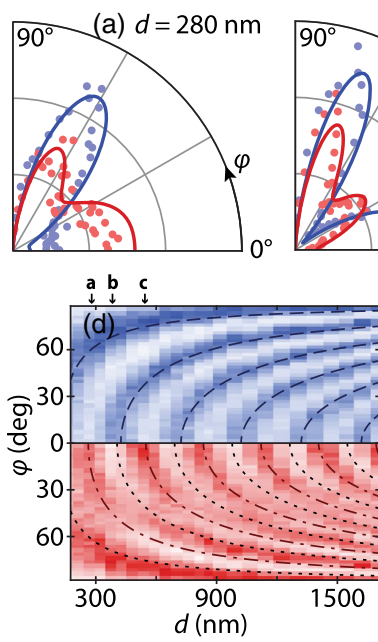

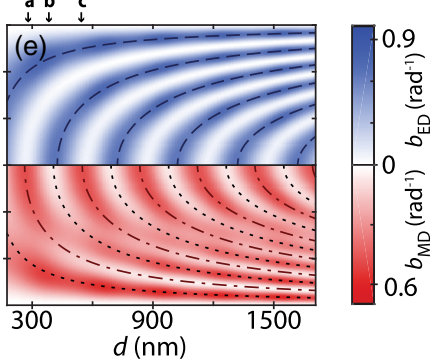

FIG. 3. (a)-(c) SPP radiation patterns of isotropic ED (blue) and MD sources (red) at different distances $d$ from a plasmonic reflector. (Dots) Experimental data; (Lines) Calculated patterns. (d) Experimental and (e) calculated angular ED and MD branching ratios as color maps. Analytically calculated self-interference maxima for $p_{z}$ (dashed), $m_{x}$ (dotted), and $m_{y}$ (dash-dotted) are overlaid. 
one around $60^{\circ}$, at the same angle as the ED maximum. The two MD lobes can be assigned to interference maxima of the dipole components $m_{y}$ and $m_{x}$, respectively, as seen when their calculated emission patterns are plotted separately (Supplemental Material Fig. S6 [22]). If $d$ is increased, the emission lobes are shifted towards larger angles and higher-order interference maxima appear at small $\varphi$ [Figs. 3(b) and 3(c)]. The emission directions of the measured radiation patterns are in good agreement with our analytical calculations (lines). The discrepancy in SPP intensity between theory and experiment at small $\varphi$ is presumably due to a distortion of the reflected SPPs at the printed line of nanocrystals and slightly stronger SPP emission along the line due to dielectric-loaded waveguiding effects.

To compare radiation patterns of subsequent measurements at different $d$ quantitatively, the emission patterns of transition $j=\{\mathrm{ED}, \mathrm{MD}\}$ can be normalized by the total SPP emission of both transitions integrated over all angles $\varphi$ to obtain angular branching ratios

$$
b_{j}(d, \varphi)=\frac{I_{j}(d, \varphi)}{\int_{0}^{\pi / 2}\left[I_{\mathrm{ED}}(d, \tilde{\varphi})+I_{\mathrm{MD}}(d, \tilde{\varphi})\right] d \tilde{\varphi}} .
$$

The normalization renders the radiation patterns independent of the number of emitters and excitation power in subsequent experiments. Figure 3(d) shows experimental $b_{\mathrm{MD}}$ (red) and $b_{\mathrm{ED}}$ (blue) values as a color map, constructed from a series of measurements on nanocrystals printed at increasing distance $d$ from the reflector. For $b_{\mathrm{ED}}$, an alternating pattern with maxima and minima is observed, stemming from constructive and destructive selfinterference. $b_{\mathrm{MD}}$ features a more complicated pattern, dominated by $m_{x}$ at $\varphi$ larger than $45^{\circ}$ and by $m_{y}$ at $\varphi$ smaller than $45^{\circ}$, respectively. This can qualitatively be understood from the intensity scaling factors $\sin ^{2} \varphi$ and $\cos ^{2} \varphi$ in Eqs. (2) and (3). This is further verified by overlaying curves representing analytically calculated selfinterference maxima of $p_{z}$ (dashed), $m_{x}$ (dotted), and $m_{y}$ (dash-dotted). Those curves match the locations with high angular branching ratios of the corresponding transitions. The agreement between experiment [Fig. 3(d)] and theory [Fig. 3(e)] indicates that the effects responsible for the directional SPP emission for both MD and ED emitters are well captured by our simple selfinterference model.

Not only the SPP emission direction, but also the corresponding SPP emission rates through the ED and MD transitions are affected by the local plasmonic environment around the dipole sources, as seen in Fig. 4. We plot the fraction of SPPs launched over all $\varphi$ by relaxation through the transition $j$ for a source at a given $d$, i.e., the branching ratio of transition $j$,

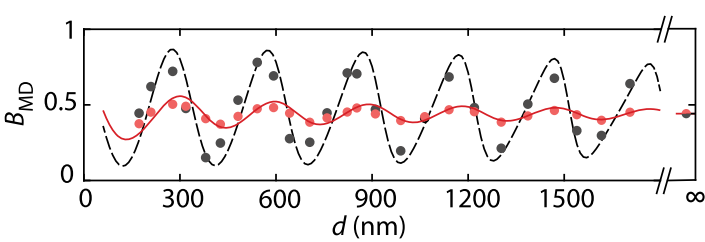

FIG. 4. Experimental (dots) and calculated (lines) MD branching ratios as function of emitter-reflector separation $d$, integrated over all SPP emission angles (red). The narrow-angle branching ratio depicted in black only considers emission angles $\varphi$ smaller than $10^{\circ}$.

$$
B_{j}(d)=\frac{\int_{0}^{\pi / 2} I_{j}(d, \varphi) d \varphi}{\int_{0}^{\pi / 2}\left[I_{\mathrm{ED}}(d, \varphi)+I_{\mathrm{MD}}(d, \varphi)\right] d \varphi} .
$$

The branching ratio of the MD transition, $B_{\mathrm{MD}}=1-B_{\mathrm{ED}}$, oscillates around 0.44 (red line and dots in Fig. 4). This value corresponds to the branching ratio without the plasmonic reflector [Figs. 1(c)-1(e)]. The oscillation period of $\sim 295 \mathrm{~nm}$ is on the order of half the SPP wavelength, slightly influenced by the difference in the ED and MD emission wavelengths. A modulation of $B_{\mathrm{MD}}$ between $\sim 0.35$ and $\sim 0.5$ is measured at close emitter-reflector distances. Hence, not only the direction of the SPP emission is affected by the plasmonic environment, but also the angle-integrated branching ratios of the ED and MD transitions. When increasing the emitter-reflector distance, the branching ratio flattens out. The slight difference in ED and MD emission wavelength superposes an additional beating pattern (Supplemental Material Fig. S7 [22]).

The modulation of the MD branching ratio due to selfinterference is even larger if we consider only SPPs emitted perpendicular to the reflector, i.e., at $\varphi$ close to zero. For those angles, SPP emission from the $m_{x}$ component can be neglected because of the $\sin ^{2} \varphi$ dependence in Eq. (2). The opposing field symmetry of the remaining dipole components $m_{y}$ and $p_{z}$ yields a large modulation of the narrowangle branching ratio $B_{\mathrm{MD}}^{\varphi \leq 10^{\circ}}$, here defined as in Eq. (6) but with upper integration bounds of $10^{\circ}$. As seen in Fig. 4 (black dashed line and circles), $B_{\mathrm{MD}}^{\varphi \leq 10^{\circ}}$ oscillates between $\sim 0.8$ and $\sim 0.15$; i.e., the SPP emission at small angles via the MD transition can selectively be tuned between four times stronger and five times weaker than the corresponding ED emission intensity. This could be used to investigate weak ED-forbidden transitions in quantum emitters [32], hidden by stronger transitions, through spatial separation of their SPP emission. Alternatively, curved reflectors could be designed that selectively enhance only one of the transitions for all emission angles. Furthermore, by reciprocity, a selective excitation through either MD or ED transitions of quantum emitters [33] or MD or ED moments of scatterers by SPPs incoming only from certain angles should be possible.

The branching ratio $B_{j}$ describes the competition between ED and MD transitions from a common excited 
state for a given emitter (here $\mathrm{Eu}^{3+}$ in $\mathrm{NaYF}_{4}$ ). In analogy to Ref. [21], we can divide $I_{j}, I_{\mathrm{ED}}$, and $I_{\mathrm{MD}}$ in Eq. (6) by the oscillator strengths of the corresponding transitions to obtain the relative magnetic $(j=\mathrm{MD})$ or electric $(j=\mathrm{ED})$ local density of optical states (LDOS) associated with

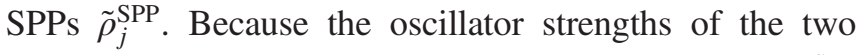
considered transitions of our emitters are very similar, $\tilde{\rho}_{\mathrm{MD}}^{\mathrm{SPP}}$ (Supplemental Material Fig. S8 [22]) closely resembles $B_{\mathrm{MD}}$ (Fig. 4). Therefore, the relative LDOS associated with SPPs features oscillations in our two-dimensional experiment for SPPs. This is distinctively different from previous three-dimensional experiments for dipole sources in front of a photonic mirror (see discussion and Supplemental Material Figs. S9(a)-S9(c) vs Figs. S9(d)-S9(f) in Ref. [22]). There, the oscillations are due to photonic contributions to the LDOS $[13,17,19]$. The coupling of ED and MD sources to SPPs both decrease exponentially with source-mirror separation in the three-dimensional experiment, leading to a separation-independent $\tilde{\rho}_{\mathrm{MD}}^{\mathrm{SPP}}$ [Supplemental Material Fig. S9(c) [22]] qualitatively different from the oscillations that we find here [Supplemental Material Figs. S8 and S9(f) [22] ].

To summarize, we have experimentally mapped selfinterference effects of electric- and magnetic-dipole sources placed with subwavelength precision in proximity to a plasmonic reflector. The presented method using $\mathrm{Eu}^{3+}$ doped nanocrystals as a probe can readily be adapted to investigate the electromagnetic environment around more complex plasmonic and photonic structures, such as curved reflectors or optical antennas. Our results highlight the importance of differing SPP field symmetries of ED and MD sources for the interaction with their plasmonic environment. The findings are relevant for a rational design of the plasmonic environment around sources of SPPs, in particular, for lanthanide or transition-metal ions that exhibit MD transitions and for plasmonic scatterers, such as pillars or holes, with significant magnetic polarizability.

This research was supported by the European Research Council under the European Union's Seventh Framework Programme (FP/2007-2013) through ERC Grant Agreement No. 339905 (QuaDoPS Advanced Grant). P. R. is supported by the Swiss National Science Foundation under Grant No. 200021_146180. F. T. R. and B. 1. F. acknowledge support from The Netherlands Organisation for Scientific Research (NWO Rubicon Grants No. 680-50-1509 and No. 680-50-1513, respectively).

*Corresponding author. dnorris@ethz.ch

[1] A. V. Akimov, A. Mukherjee, C. L. Yu, D. E. Chang, A. S. Zibrov, P. R. Hemmer, H. Park, and M. D. Lukin, Generation of single optical plasmons in metallic nanowires coupled to quantum dots, Nature (London) 450, 402 (2007).
[2] S. J. P. Kress, P. Richner, S. V. Jayanti, P. Galliker, D. K. Kim, D. Poulikakos, and D. J. Norris, Near-field light design with colloidal quantum dots for photonics and plasmonics, Nano Lett. 14, 5827 (2014).

[3] M. Berthel, Q. Jiang, A. Pham, J. Bellessa, C. Genet, S. Huant, and A. Drezet, Directional Local Density of States of Classical and Quantum Propagating Surface Plasmons, Phys. Rev. Applied 7, 014021 (2017).

[4] B. Hecht, H. Bielefeldt, L. Novotny, Y. Inouye, and D. W. Pohl, Local Excitation, Scattering, and Interference of Surface Plasmons, Phys. Rev. Lett. 77, 1889 (1996).

[5] S.-Y. Lee, I.-M. Lee, J. Park, S. Oh, W. Lee, K.-Y. Kim, and B. Lee, Role of Magnetic Induction Currents in Nanoslit Excitation of Surface Plasmon Polaritons, Phys. Rev. Lett. 108, 213907 (2012).

[6] N. Rotenberg, M. Spasenović, T. L. Krijger, B. le Feber, F. J. G. de Abajo, and L. Kuipers, Plasmon Scattering from Single Subwavelength Holes, Phys. Rev. Lett. 108, 127402 (2012).

[7] M. Kuttge, E. J. R. Vesseur, A. F. Koenderink, H. J. Lezec, H. A. Atwater, F. J. G. de Abajo, and A. Polman, Local density of states, spectrum, and far-field interference of surface plasmon polaritons probed by cathodoluminescence, Phys. Rev. B 79, 113405 (2009).

[8] D. Zhu, Z. Dong, H.-S. Chu, Y. A. Akimov, and J. K. W. Yang, Image dipole method for the beaming of plasmons from point sources, ACS Photonics 1, 1307 (2014).

[9] S. J. P. Kress, J. Cui, P. Rohner, D. K. Kim, F. V. Antolinez, K.-A. Zaininger, S. V. Jayanti, P. Richner, K. M. McPeak, D. Poulikakos, and D. J. Norris, A customizable class of colloidal-quantum-dot spasers and plasmonic amplifiers, Sci. Adv. 3, e1700688 (2017).

[10] T. Coenen and A. Polman, Optical properties of single plasmonic holes probed with local electron beam excitation, ACS Nano 8, 7350 (2014).

[11] I. Sersic, M. Frimmer, E. Verhagen, and A. F. Koenderink, Electric and Magnetic Dipole Coupling in Near-Infrared Split-Ring Metamaterial Arrays, Phys. Rev. Lett. 103, 213902 (2009).

[12] A. I. Kuznetsov, A. E. Miroshnichenko, Y. H. Fu, J. Zhang, and B. Luk'yanchuk, Magnetic light, Sci. Rep. 2, 492 (2012).

[13] F. T. Rabouw, P. T. Prins, and D. J. Norris, Europium-doped $\mathrm{NaYF}_{4}$ nanocrystals as probes for the electric and magnetic local density of optical states throughout the visible spectral range, Nano Lett. 16, 7254 (2016).

[14] D. Li, M. Jiang, S. Cueff, C. M. Dodson, S. Karaveli, and R. Zia, Quantifying and controlling the magnetic dipole contribution to $1.5-\mu \mathrm{m}$ light emission in erbium-doped yttrium oxide, Phys. Rev. B 89, 161409 (2014).

[15] B. Choi, M. Iwanaga, Y. Sugimoto, K. Sakoda, and H. T. Miyazaki, Selective plasmonic enhancement of electric- and magnetic-dipole radiations of Er ions, Nano Lett. 16, 5191 (2016).

[16] K. H. Drexhage, H. Kuhn, and F. P. Schäfer, Variation of the fluorescence decay time of a molecule in front of a mirror, Ber. Bunsen-Ges. Phys. Chem. 72, 329 (1968).

[17] K. H. Drexhage, Influence of a dielectric interface on fluorescence decay time, J. Lumin. 1-2, 693 (1970).

[18] W. L. Barnes, Fluorescence near interfaces: the role of photonic mode density, J. Mod. Opt. 45, 661 (1998). 
[19] S. Karaveli and R. Zia, Spectral Tuning by Selective Enhancement of Electric and Magnetic Dipole Emission, Phys. Rev. Lett. 106, 193004 (2011).

[20] T. H. Taminiau, S. Karaveli, N. F. van Hulst, and R. Zia, Quantifying the magnetic nature of light emission, Nat. Commun. 3, 979 (2012).

[21] L. Aigouy, A. Cazé, P. Gredin, M. Mortier, and R. Carminati, Mapping and Quantifying Electric and Magnetic Dipole Luminescence at the Nanoscale, Phys. Rev. Lett. 113, 076101 (2014).

[22] See Supplemental Material at http://link.aps.org/ supplemental/10.1103/PhysRevLett.121.113601, which includes Refs. [23-25], for technical details of the fabrication of the plasmonic sample, synthesis of $\mathrm{NaYF}_{4}: \mathrm{Eu}^{3+}$ nanocrystals, electrohydrodynamic nanoprinting, spectroscopic experiments, details of the analytical self-interference model, and a comparison of this work with previous experiments.

[23] Z. Li and Y. Zhang, An efficient and user-friendly method for the synthesis of hexagonal-phase $\mathrm{NaYF}_{4}: \mathrm{Yb}, \mathrm{Er} / \mathrm{Tm}$ nanocrystals with controllable shape and upconversion fluorescence, Nanotechnology 19, 345606 (2008).

[24] F. Wang, R. Deng, and X. Liu, Preparation of core-shell $\mathrm{NaGdF}_{4}$ nanoparticles doped with luminescent lanthanide ions to be used as upconversion-based probes, Nat. Protoc. 9, 1634 (2014).

[25] J. Schneider, P. Rohner, D. Thureja, M. Schmid, P. Galliker, and D. Poulikakos, Electrohydrodynamic NanoDrip printing of high aspect ratio metal grid transparent electrodes, Adv. Funct. Mater. 26, 833 (2016).
[26] P. Nagpal, N. C. Lindquist, S.-H. Oh, and D. J. Norris, Ultrasmooth patterned metals for plasmonics and metamaterials, Science 325, 594 (2009).

[27] K. M. McPeak, S. V. Jayanti, S. J. P. Kress, S. Meyer, S. Iotti, A. Rossinelli, and D. J. Norris, Plasmonic films can easily be better: Rules and recipes, ACS Photonics 2, 326 (2015).

[28] P. Galliker, J. Schneider, H. Eghlidi, S. Kress, V. Sandoghdar, and D. Poulikakos, Direct printing of nanostructures by electrostatic autofocussing of ink nanodroplets, Nat. Commun. 3, 890 (2012).

[29] R. E. Kunz and W. Lukosz, Changes in fluorescence lifetimes induced by variable optical environments, Phys. Rev. B 21, 4814 (1980).

[30] G. W. Ford and W. H. Weber, Electromagnetic interactions of molecules with metal surfaces, Phys. Rep. 113, 195 (1984).

[31] L. Novotny and B. Hecht, Principles of Nano-Optics, 2nd ed (Cambridge University Press, Cambridge, England, 2012).

[32] Y. Zhou, G. Scuri, D. S. Wild, A. A. High, A. Dibos, L. A. Jauregui, C. Shu, K. De Greve, K. Pistunova, A. Y. Joe, T. Taniguchi, K. Watanabe, P. Kim, M. D. Lukin, and H. Park, Probing dark excitons in atomically thin semiconductors via near-field coupling to surface plasmon polaritons, Nat. Nanotechnol. 12, 856 (2017).

[33] M. Kasperczyk, S. Person, D. Ananias, L. D. Carlos, and L. Novotny, Excitation of Magnetic Dipole Transitions at Optical Frequencies, Phys. Rev. Lett. 114, 163903 (2015). 